Case Report

\title{
Vaginal Evisceration: An Unexpected Complication of Conization
}

\author{
Ali Ghassani, ${ }^{1}$ Benoit Andre, ${ }^{1,2}$ Caroline Simon-Toulza, \\ Yann Tanguy le Gac, ${ }^{1}$ Alejandra Martinez, ${ }^{2}$ and Fabien Vidal ${ }^{3}$ \\ ${ }^{1}$ CHU Toulouse, Pole de Gynécologie Obstétrique, Hôpital Paule de Viguier, 31059 Toulouse, France \\ ${ }^{2}$ Surgery Department, Institut Claudius Regaud, 20-24 rue du pont Saint Pierre, 31052 Toulouse, France \\ ${ }^{3}$ Hôpital Paule de Viguier-CHU Toulouse, Service de Chirurgie Gynécologique, Pole de Gynécologie Obstétrique, \\ 31059 Toulouse Cedex 9, France
}

Correspondence should be addressed to Fabien Vidal; dox_94@yahoo.fr

Received 19 July 2014; Accepted 6 November 2014; Published 19 November 2014

Academic Editor: Loïc Sentilhes

Copyright ( 2014 Ali Ghassani et al. This is an open access article distributed under the Creative Commons Attribution License, which permits unrestricted use, distribution, and reproduction in any medium, provided the original work is properly cited.

\begin{abstract}
Background. Large loop excision of the transformation zone (LLETZ) is routinely performed for the management of high grade intracervical neoplasia (CIN). Several uncommon complications have been described, including postoperative peritonitis, pseudoaneurysm of uterine artery, and bowel fistula. We report a unique case of postoperative vaginal evisceration and the subsequent management. Case. A 73-years-old woman underwent LLETZ for high grade CIN. On postoperative day 3, she was admitted for small bowel evisceration through the vagina. Surgical management was based on combined laparoscopic and transvaginal approach and consisted in bowel inspection and reinstatement, peritoneal washing, and dehiscence repair. Conclusions. Vaginal evisceration is a rare but potentially serious complication of pelvic surgery. This case report is to make clinicians aware of such complication following LLETZ and its management.
\end{abstract}

\section{Introduction}

Conization is the mainstay of treatment for high grade cervical intraepithelial neoplasia (CIN). Three different excisional methods (cold knife conization, loop electrosurgical excision, and laser conization) are available and, to date, none of them seem superior to another [1]. Large loop excision of the transformation zone (LLETZ) is a common surgical procedure using a small wire loop and electrical current, with recognized complications such as bleeding, infection, and postoperative cervical stenosis [2]. It is also associated with poorer obstetrical outcomes, including increased rates of preterm delivery and perinatal mortality [2]. Most procedures are performed under local anesthesia with ambulatory care.

Herein we report the first case to our knowledge of acute vaginal evisceration following LLETZ.

\section{Observation}

A 73-years-old woman was referred to our institution for the management of high grade intraepithelial neoplasia confirmed by colposcopy-guided biopsy. LLETZ procedure was performed under general anesthesia and ended with monopolar coagulation of the posterior part of the resection because of arterial and venous bleeding. A vaginal mesh was left in place and removed before the patient was discharged, on the day of the surgery.

Three days after surgery, the patient was admitted in the emergency department for bulging vagina and bowel obstructive syndrome. She had no history of postoperative bleeding or pain. Clinical examination revealed small bowel evisceration through the vagina. Bowel loops were edematous but viable (Figure 1).

Evisceration was managed by a combined laparoscopic and vaginal approach. Small bowel reinstatement was 


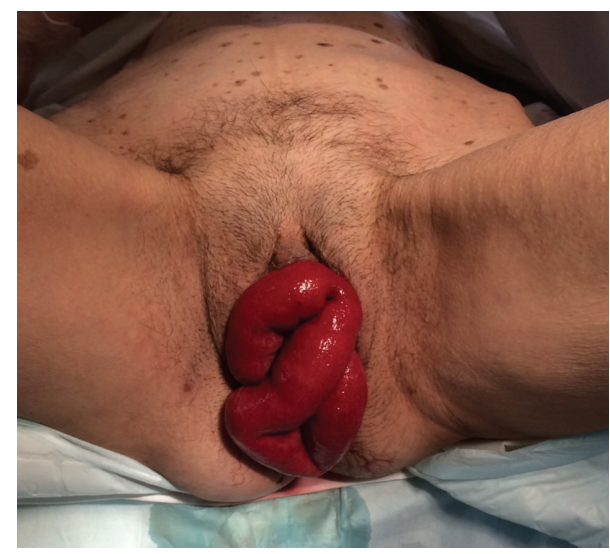

FIGURE 1: Small bowel evisceration through the vagina.

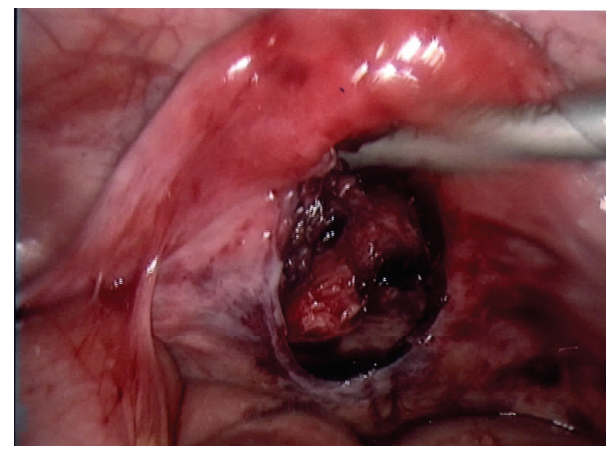

FIGURE 2: Vaginal wall defect (laparoscopic view).

performed vaginally with laparoscopic guidance and revealed a large defect in the vaginal posterior wall next to the site of LLETZ (Figure 2). Laparoscopic evaluation found neither bowel abnormalities nor any peritonitis.

Treatment consisted in peroperative antibiotic prophylaxis (amoxicillin plus clavulanic acid $2 \mathrm{~g}$ ), peritoneal washing, and vaginal repair with interrupted laparoscopic Vicryl sutures (Figure 3). Procedure duration was $90 \mathrm{~min}$.

The patient recovered uneventfully and was discharged on 4th postoperative day. Delayed follow-up was normal.

\section{Discussion}

In this report, we introduce the first published case of vaginal evisceration following LLETZ. It was managed by a combined vaginal and laparoscopic approach and consisted in bowel reinstatement, peritoneal washing, and vaginal repair.

Vaginal evisceration is a rare but potentially life threatening complication. Its incidence after any type of pelvic surgery is $0.03 \%[3,4]$. Major risk factors are postmenopausal state, increased abdominal pressure, and hysterectomy [5]. Among patients who undergo hysterectomy, laparoscopic route is associated with a higher incidence of cuff dehiscence, compared to transvaginal and abdominal procedures [6].

LLETZ is a common surgical procedure for the management of CIN with recognized complications. Several

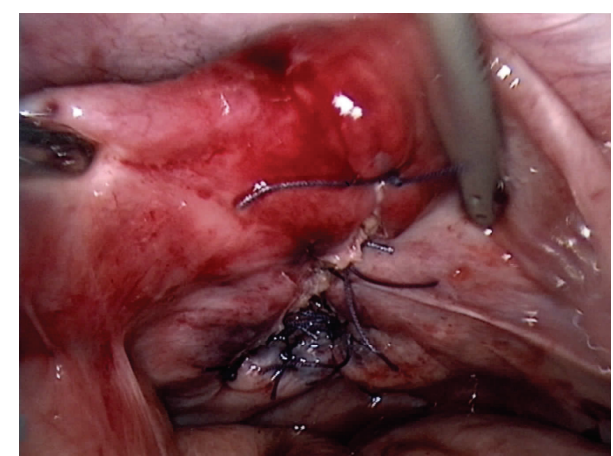

FIGURE 3: Laparoscopic repair of the defect involving Vicryl simple suture.

uncommon complications of conization have been described including fistula formation [7], intestinal occlusion [8], intraabdominal hemorrhage [9], pseudoaneurysm of uterine artery [10], peritonitis [11], retroperitoneal hematoma [12], uterine avulsion [13], and extrapelvic abscesses [14].

Vaginal evisceration following LLETZ is quite surprising since the vagina is not concerned by the resection. Etiologic mechanism may thus involve an unrecognized iatrogenic posterior colpotomy or necrosis of the posterior vaginal fornix due to monopolar coagulation. Similar event has been previously reported by Varras et al. [11] but led to infectious peritonitis rather than evisceration.

Morbidity associated with vaginal evisceration is high: $15 \%$ of patients develop postoperative complications and $20 \%$ require bowel resection [15]. Delay in diagnosis and treatment increases the risk of bowel infarction. Hence its management is a medical emergency.

Surgery is the mainstay of treatment. Concomitant intravenous administration of antibiotics is recommended due to bowel extraperitoneal exposure [11, 15-17]. Several routes of surgery have been described: vaginal, abdominal, or combined $[5,17,18]$. Regarding abdominal route, former laparotomy approach has been replaced by laparoscopy whenever applicable [15]. According to a review focusing on vaginal evisceration following hysterectomy, surgical approaches are mostly vaginal or abdominal while only $15 \%$ of the patients benefit from a combined approach [5]. We strongly recommend the use of such combined surgery that allows excluding associated and unrecognized bowel injuries and washing properly the peritoneal cavity [19-21]. It should be systematically performed in patients who might have poor tissue quality (postmenopausal, gynecologic malignancies, history of pelvic radiation therapy).

In most cases, the surgical management of vaginal defect consists in a primary repair involving simple suture. Some authors have also described techniques using synthetic mesh or omental flap to strengthen vaginal repair $[15,22,23]$.

The prevention of such complication is another important issue, although the etiologic mechanism remains unclear in our case. Nevertheless, caution should be paid in the use of monopolar coagulation, particularly in atrophic tissues. Bipolar energy or hemostatic sutures should be preferred for persistent and severe bleeding. To avoid unintentional 
colpotomy, a soft pulling should be exerted on the cervix during LLETZ. Last, whenever a peroperative complication such as hemorrhage occurs, the use of vaginal retractors may improve exposure and subsequent management. Indeed, vaginal dehiscence can be managed with minimal morbidity if recognized immediately.

\section{Conclusion}

Vaginal evisceration is a rare but potentially serious complication of pelvic surgery. Most cases were described after hysterectomy. We introduce here the first report to our knowledge of such complication after conization.

Vaginal evisceration is a surgical emergency, since delay in treatment exposes to increased morbimortality. Our management was based on a combined laparoscopic and transvaginal approach leading to favorable outcomes. Although our single case report does not provide a strong level of evidence, we highly recommend this surgical attitude for the management of such complication.

\section{Conflict of Interests}

The authors do not have any conflict of interests in this study.

\section{References}

[1] P. P. L. Martin-Hirsch, E. Paraskevaidis, A. Bryant, and H. O. Dickinson, "Surgery for cervical intraepithelial neoplasia," The Cochrane Database of Systematic Reviews, vol. 12, Article ID CD001318, 2013.

[2] J. J. Baldauf, E. Baulon, V. Thoma, A. S. Woronoff, and C. Y. Akladios, "Conséquences obstétricales des conisations à l'anse diathermique et facteurs aggravants," Journal de Gynécologie Obstétrique et Biologie de la Reproduction, vol. 42, no. 6, pp. 534540, 2013.

[3] A. J. Croak, J. B. Gebhart, C. J. Klingele, G. Schroeder, R. A. Lee, and K. C. Podratz, "Characteristics of patients with vaginal rupture and evisceration," Obstetrics and Gynecology, vol. 103, no. 3, pp. 572-576, 2004.

[4] P. D. Iaco, M. Ceccaroni, C. Alboni et al., “Transvaginal evisceration after hysterectomy: is vaginal cuff closure associated with a reduced risk?" European Journal of Obstetrics \& Gynecology and Reproductive Biology, vol. 125, no. 1, pp. 134-138, 2006.

[5] P. T. Ramirez and D. P. Klemer, "Vaginal evisceration after hysterectomy: a literature review," Obstetrical \& Gynecological Survey, vol. 57, no. 7, pp. 462-467, 2002.

[6] M. Wallwiener, F. A. Taran, R. Rothmund et al., "Laparoscopic supracervical hysterectomy (LSH) versus total laparoscopic hysterectomy (TLH): an implementation study in 1,952 patients with an analysis of risk factors for conversion to laparotomy and complications, and of procedure-specific re-operations," Archives of Gynecology and Obstetrics, vol. 288, no. 6, pp. 13291339, 2013.

[7] H. Krissi, T. Levy, Z. Ben-Rafael, and H. Levavi, "Fistula formation after large loop excision of the transformation zone in patients with cervical intraepithelial neoplasia," Acta Obstetricia et Gynecologica Scandinavica, vol. 80, no. 12, pp. 1137-1138, 2001.

[8] P. Raynal and J. Lucas, "Pelviperitonitis and intestinal occlusion: two unusual cold-knife conisation complications," Journal de
Gynecologie Obstetrique et Biologie de la Reproduction, vol. 32, no. 1, pp. 43-45, 2003.

[9] P. Nannapaneni, R. Naik, A. de Barros Lopes, and J. M. Monaghan, "Intra-abdominal bleed following LLETZ," Journal of Obstetrics and Gynaecology, vol. 22, no. 1, pp. 99-100, 2002.

[10] J. Jain, S. O'Leary, and M. Sarosi, "Uterine artery pseudoaneurysm after uterine cervical conization," Obstetrics and Gynecology, vol. 123, no. 2, part 2, pp. 456-458, 2014.

[11] M. Varras, C. Akrivis, A. Anastasiadis, G. Lekkas, and G. Diakakis, "Peritonitis due to iatrogenic colpotomy after large loop excision of the transformation zone (LLETZ) in a patient with cervical intraepithelial neoplasia III: our experience of a rare case with review of the literature," European Journal of Gynaecological Oncology, vol. 33, no. 2, pp. 214-216, 2012.

[12] C. F. Brown, I. S. Mashini, W. A. Turner, and D. G. Gallup, "Retroperitoneal hematoma: an unusual complication of cold knife conization of the cervix," Obstetrics \& Gynecology, vol. 68, no. 3, pp. 66S-67S, 1986.

[13] S. Santos-Ribeiro, A. Coelho, T. D. da Costa et al., "Laparoscopic re-anastomosis of a uterine avulsion following cold-knife conization," Surgical Technology International, vol. 24, pp. 231235, 2014.

[14] A. D. Treszezamsky, M. F. M. Boero, and I. Mehta, "Cervical conization complicated by sepsis with lung and liver abscesses," Journal of Lower Genital Tract Disease, vol. 14, no. 2, pp. 130-133, 2010.

[15] F. Narducci, Y. Sonoda, E. Lambaudie, E. Leblanc, and D. Querleu, "Vaginal evisceration after hysterectomy: the repair by a laparoscopic and vaginal approach with a omental flap," Gynecologic Oncology, vol. 89, no. 3, pp. 549-551, 2003.

[16] M. D. Sinclair, A. R. Davies, S. Sankaran, S. Agnihotri, and S. M. Andreani, "Laparoscopic repair of spontaneous vaginal evisceratlon of small bowel: report of a case," Annals of the Royal College of Surgeons of England, vol. 92, no. 1, pp. W3-W5, 2010.

[17] M. D. Moen, M. Desai, and R. Sulkowski, "Vaginal evisceration managed by transvaginal bowel resection and vaginal repair," International Urogynecology Journal and Pelvic Floor Dysfunction, vol. 14, no. 3, pp. 218-220, 2003.

[18] I. Nikolopoulos, H. Khan, G. Janakan, and R. Kerwat, "Laparoscopically assisted repair of vaginal evisceration after hysterectomy," BMJ Case Reports, 2013.

[19] C. H. Nezhat, F. Nezhat, and D. S. Seidman, "Vaginal vault evisceration after total laparoscopic hysterectomy," Obstetrics \& Gynecology, vol. 87, no. 5, part 2, pp. 868-870, 1996.

[20] R. E. Symmonds, J. H. Pratt, and F. Henry Ellis Jr., "Ruptured enterocele," American Journal of Obstetrics and Gynecology, vol. 74, no. 5, pp. 1150-1153, 1957.

[21] R. J. Codd, B. Scourfield, S. Chakravarthy, and G. L. Williams, "Small bowel trans-vaginal evisceration following vault biopsy: general surgeons beware!," Annals of the Royal College of Surgeons of England, vol. 92, no. 7, pp. W6-W7, 2010.

[22] D. Jurus, P. Finamore, and B. Vakili, "Use of synthetic mesh to prevent recurrent vaginal evisceration: a case report," International Urogynecology Journal and Pelvic Floor Dysfunction, vol. 20, no. 2, pp. 259-260, 2009.

[23] C. Crespi and A. M. de Giorgio, "Prolapse of the vaginal vault complicated by ileal evisceration. Possible role of dacron mesh in emergencies. Technical notes," Giornale di Chirurgia, vol. 12, no. 10 , pp. 498-500, 1991. 


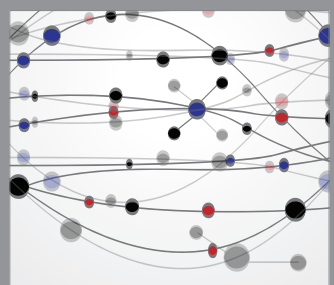

The Scientific World Journal
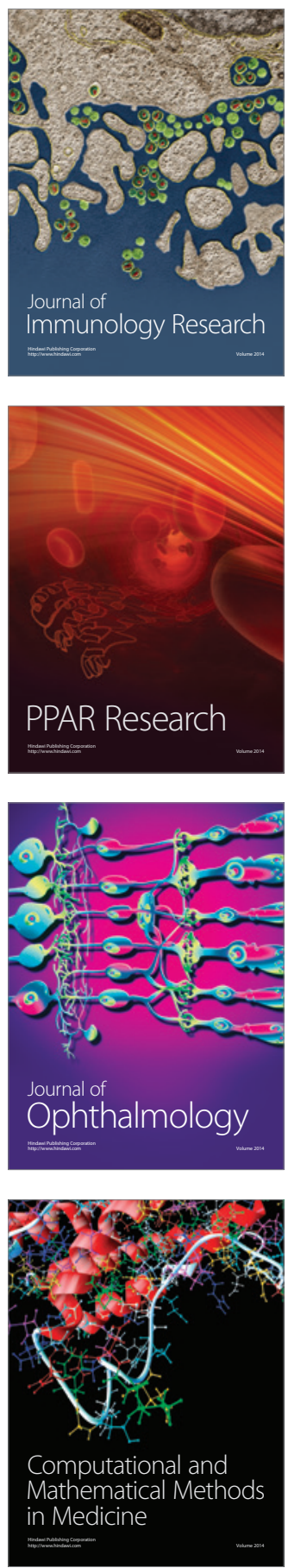

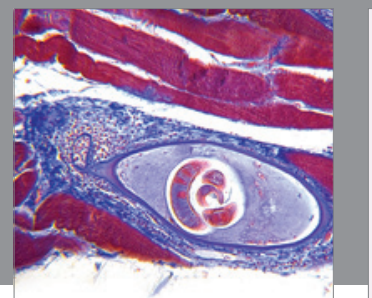

Gastroenterology

Research and Practice
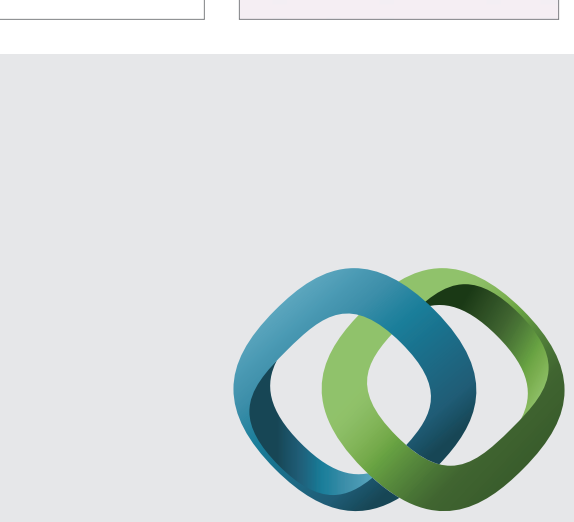

\section{Hindawi}

Submit your manuscripts at

http://www.hindawi.com
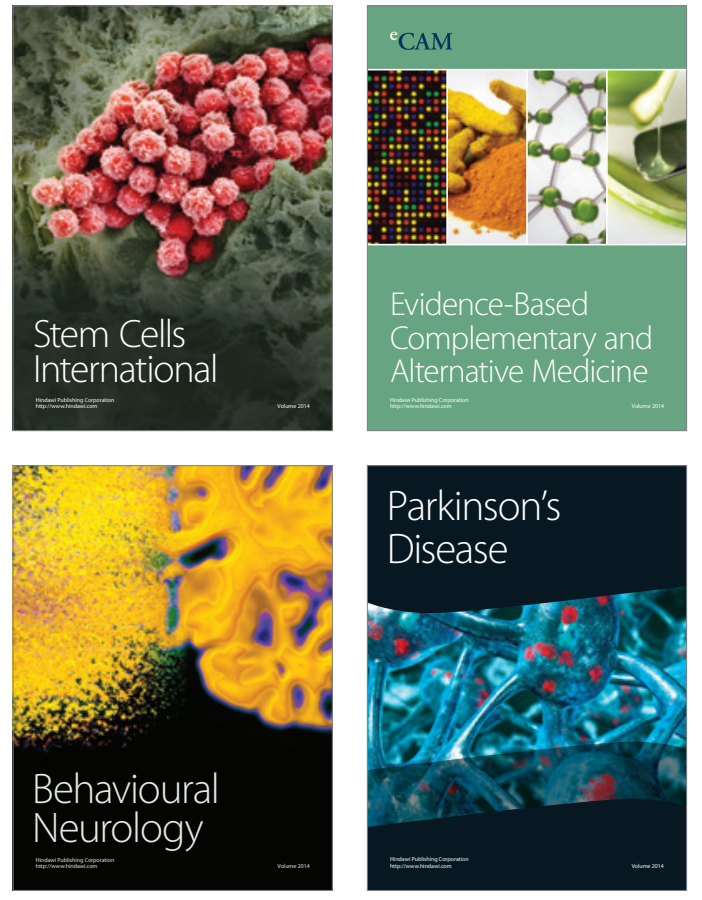
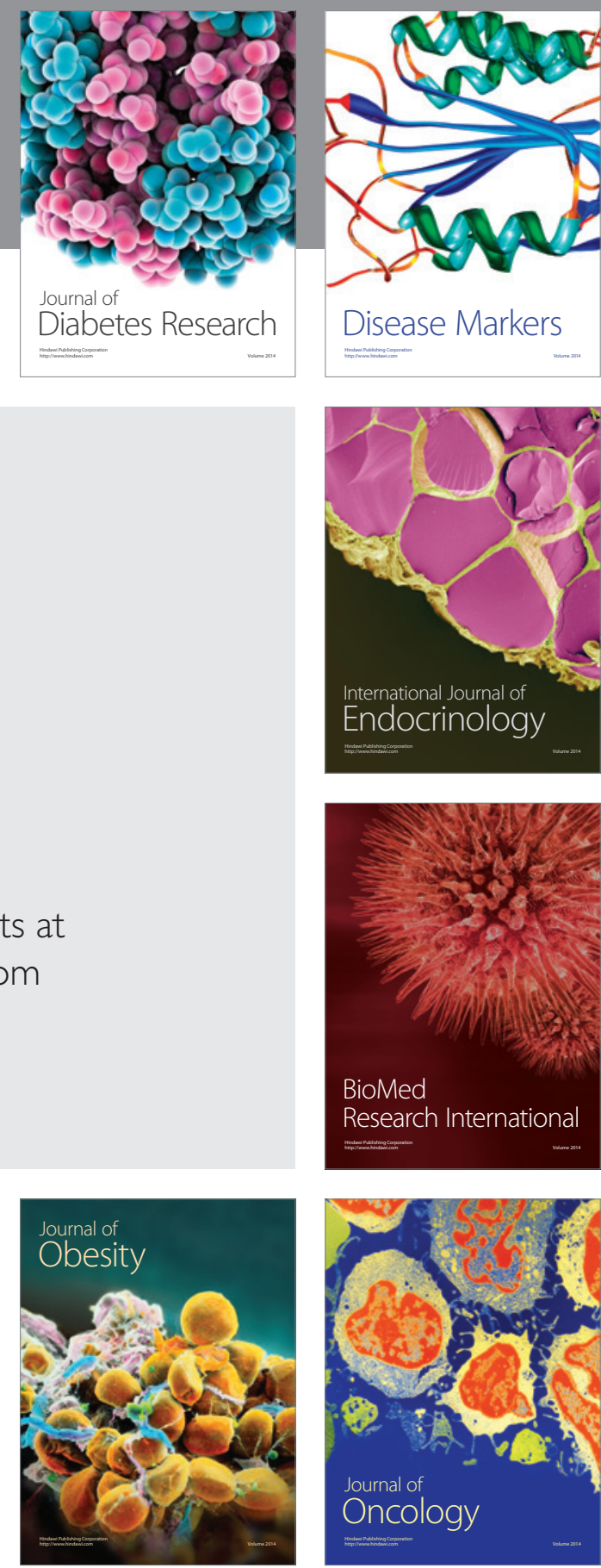

Disease Markers
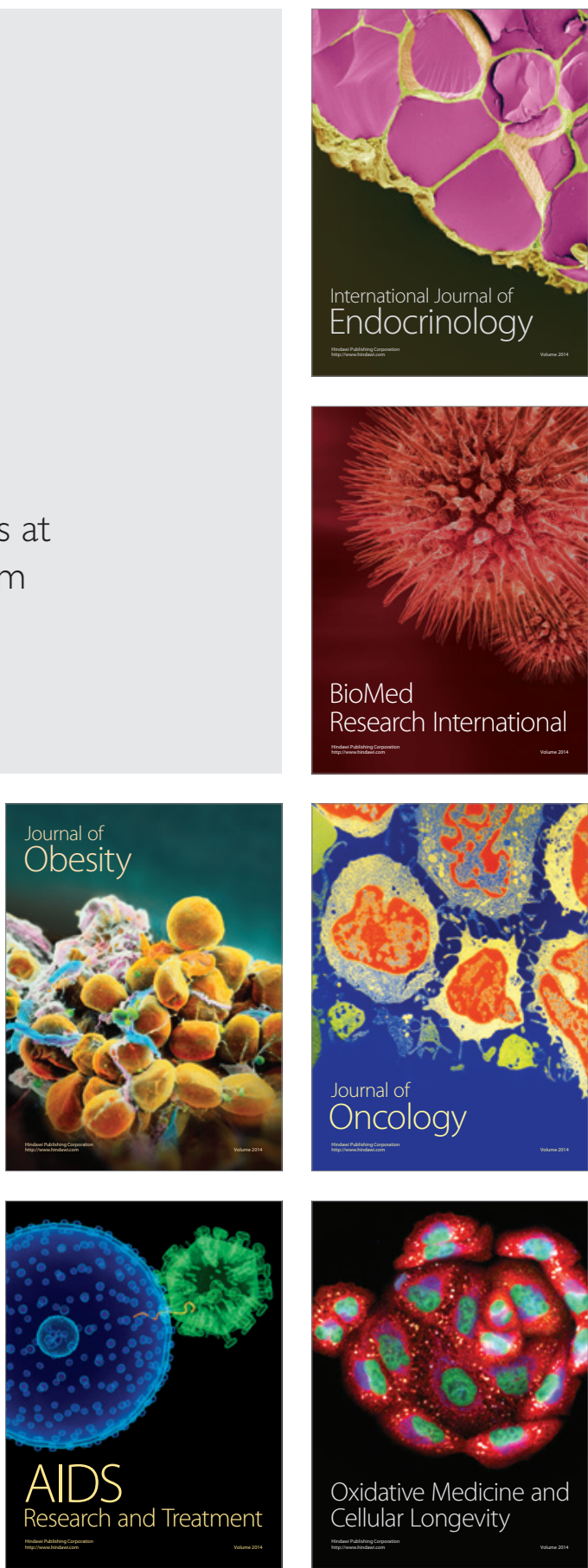